\title{
Robinson-Trautman Equations and Chandrasekhar's Special Perturbation of the Schwarzschild Metric
}

\author{
Guoying $\mathrm{Qi}^{1,2}$ and Bernard F. Schutz ${ }^{1}$ \\ Received April 14, 1993
}

\begin{abstract}
A perturbation wave solution of the Robinson-Trautman equations is proved to be a perturbation of the Schwarzschild black hole which describes an outgoing axial gravitational wave and corresponds to a special case of Chandrasekhar's algebraically special perturbation of the Schwarzschild metric.
\end{abstract}

Gravitational perturbations of the Schwarzschild black hole have been studied for more than twenty years [1]. Recently much research has focused on the equations given by Regge and Wheeler [2] and by Zerilli [3], and in particular on the quasi-normal mode solutions of these equations [4-7]. The nature of the potential in the equations describing the perturbation makes it difficult to obtain exact analytical solutions, so that most work has employed numerical methods of one kind or another [4-7]. The only exact analytical perturbation solution known was found by Chandrasekhar [8], and Leaver [5] pointed out that it seemed to be part of a sequence of quasi-normal modes. It has, uniquely among the quasi-normal modes, a purely imaginary frequency.

Recent numerical computations [7,9], however, have not given a quasinormal mode with exactly the purely imaginary frequency that corre-

1 Department of Physics and Astronomy, University of Wales College of Cardiff, Cardiff CF2 3YB, UK

2 Physics Department, Liaoning Normal University, Dalian 116022 P.R. China 
sponds to the Chandrasekhar solution. This raises many questions about the nature of the solution: Is it really a mode? What is special about its frequency? Chandrasekhar found the solution by demanding that the Starobinsky constant vanish; are there other ways of finding it?

In this paper we show that Chandrasekhar's special solution is simply the linear approximation to the Robinson-Trautman (RT) solution [10] where that solution limits to the Schwarzschild solution. It describes a purely outgoing axial wave.

The Robinson-Trautman metric [11] (with signature -2)

$$
d s^{2}=2 H d u^{2}+2 d u d r-r^{2} P^{-2} d \zeta d \bar{\zeta},
$$

where $u$ is an outgoing null coordinate, satisfies the field equations

$$
\Delta \Delta(\ln P)+12 m(\ln P)_{, u}=0
$$

and

$$
2 H=\Delta(\ln P)-2 r(\ln P)_{, u}-\frac{2 m}{r}
$$

where

$$
\Delta \equiv 4 P^{2} \cdot \partial_{\zeta} \partial_{\bar{\zeta}}
$$

In contrast to Ref. 11, we take

$$
\begin{aligned}
& \zeta=\exp (i \phi) \cot \frac{\theta}{2}, \\
& \bar{\zeta}=\exp (-i \phi) \cot \frac{\theta}{2} .
\end{aligned}
$$

When

$$
P=P_{0}=\frac{1}{2}(1+\zeta \bar{\zeta})
$$

we get

$$
2 H=2 H_{0}=1-\frac{2 m}{r},
$$

the RT metric (1) becomes the Schwarzschild metric.

We wish to study the RT equation as perturbations about the Schwarzschild metric. If we introduce the Newman-Penrose operators $\partial$ and $\bar{\partial}$ [12], operating on a function $\eta$ of $\zeta$ and $\bar{\zeta}$ with spin weight $s$, we have

$$
\bar{\partial}=2 P_{0}^{1-s} \partial_{\zeta}\left(P_{0}^{s} \eta\right), \quad \bar{\delta} \eta=2 P_{0}^{1+s} \partial_{\bar{\zeta}}\left(P_{0}^{-s} \eta\right),
$$


for which

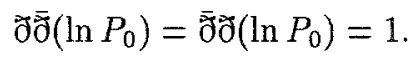

If $\eta$ has $s=0$, then we find

$$
\bar{\partial} \bar{\partial} \eta \bar{\partial} \bar{\partial} \eta=4 P_{0}^{2} \partial_{\zeta} \partial_{\bar{\zeta}} \eta .
$$

Supposing the Robinson-Trautman function $P$ can be written in the perturbation form

$$
P=P_{0} \exp \{\epsilon(u, \zeta, \bar{\zeta})\}=\frac{1}{2}(1+\zeta \bar{\zeta}) \exp \epsilon
$$

with

$$
|\epsilon| \ll 1 \text {, }
$$

the $\mathrm{RT}$ equation, eq. (2), can be rewritten as

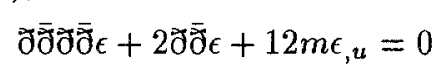

in the linear approximation. The solution of eq. (11) is

$$
\epsilon=Y_{l, m} \exp \left(-\frac{\kappa}{12 m} u\right) \text {. }
$$

It is straightforward to show that

$$
\kappa=l(l+1)[l(l+1)-2] .
$$

The coefficient $-(\kappa / 12 m)$ is just the characteristic frequency of the Chandrasekhar solution [8]. Here it is obtained in a rather natural way.

The second RT equation, eq. (3), gives

$$
2 H=1-\frac{2 m}{r}+\lambda\left(\frac{\lambda+2}{6 m} r-1\right) \epsilon
$$

where

$$
\lambda=(l-1)(l+2)
$$

Apart from $\Psi_{2}=-m r^{-3}$, the only nonvanishing Weyl scalar is

$$
\Psi_{4}=\frac{\lambda}{m r^{2}} \Theta_{l, m}[(\lambda+2) r-6 m] \exp \left\{-\frac{\lambda(\lambda+2)}{12 m} u\right\}
$$

where

$$
u=t-r_{*}
$$

and where

$$
\Theta_{l, m}=A_{l-2} Y_{i, m}+B_{l} \exp (i \phi) \cot \frac{\theta}{2}{ }_{0} Y_{l, m}
$$

with $A_{l}, B_{l}$ two normalising constants.

Equation (18) means that the solution obtained above describes an outgoing axial gravitational wave, which coincides with a special case in Ref. 8. 


\section{ACKNOWLEDGEMENTS}

We wish to thank Dr. Paul Tod for originally suggesting this investigation.

\section{REFERENCES}

1. Chandrasekhar, S. (1983). The Mathematical Theory of Black Holes (Oxford University Press, Oxford/New York).

2. Regge, T., and Wheeler, J. A. (1957). Phys. Rev. 108, 1063.

3. Zerilli, F. J. (1970). Phys. Rev. D2, 2141.

4. Schutz, B. F., and Will, C. M. (1985). Astrophys. J. 291, L33.

5. Leaver, E. W. (1986). Phys. Rev. D34, 384.

6. Sun, Y. H., and Price, R. H. (1988). Phys. Rev. D38, 1040.

7. Nollert, H.-P., and Schmidt, B. G. (1992). Phys. Rev. D45, 2617.

8. Chandrasekhar, S. (1984). Proc. Roy. Soc. Lond. A392, 1.

9. Andersson, N., and Lynnzus, S. (1992). Phys. Rev. D46, 4179.

10. Robinson, I., and Trautman, A. (1962). Proc. Roy. Soc. Lond. A265, 463.

11. Kramer, D., Stephani, H., MacCallum, M. A. H., and Herlt, E. (1980). Exact Solutions of Einstein's Field Equations (Cambridge University Press, Cambridge).

12. Newman, E. T., and Penrose, R. (1966). J. Math. Phys. 7, 863. 